\title{
Reflexive practice in interdisciplinary design of pervasive health applications in dementia care
}

\author{
Alina Huldtgren \\ Department of Media \\ University of Applied Science Düsseldorf \\ Josef-Gockeln-Str. 9, 40474 Düsseldorf \\ alina.huldtgren@fh-duesseldorf.de
}

\author{
Cordula Endter \\ Department of Cultural History and Cultural Studies \\ University of Hamburg \\ Edmund-Siemers-Allee 1, 20146 Hamburg \\ cordula.endter@uni-hamburg.de
}

\begin{abstract}
Interdisciplinary design is essential to create new pervasive health applications, in our case those for dementia care, and to innovate our healthcare systems to meet the challenges of ongoing demographic changes. While interdisciplinary design is gaining attention in HCI literature and challenges of managing and executing interdisciplinary design projects are brought to the fore, few guidelines are provided to deal with these challenges. Often published outcomes focus on the innovative solutions and describe the design process merely on a high level. However, factors that led to a certain design outcome, such as the politics of participation, power relations, understandings and negotiations of concepts, choice of settings and materials for design workshops, decisions for one design, and more importantly against another, are "black-boxed". To open the black-box and understand what kind of knowledge from who for whom and why has been created and whether and how it can be transferred to new design contexts, requires to make the above named factors visible - in publications but also during the design process. To that end we suggest a reflexive approach to interdisciplinary design through value sensitive design methods and real-time ethnographic practices.
\end{abstract}

\section{Categories and Subject Descriptors}

K.4 Computers and Society.

\section{General Terms}

Design, Human Factors.

\section{Keywords}

Dementia, co-design, interdisciplinary research, ethnography.

\section{INTRODUCTION}

Predictions state that within the next 40 years the number of people diagnosed with a form of dementia will rise from 35.6 Millions in 2010 to 114.4 Millions in 2050 [1]. Despite high investments in pharmacological research there is no successful medical cure for dementia yet. Therefore, it is important to investigate non-medical treatment and care approaches for professional and informal care that could lead to a preservation

Permission to make digital or hard copies of all or part of this work for personal or classroom use is granted without fee provided that copies are not made or distributed for profit or commercial advantage and that copies bear this notice and the full citation on the first page. To copy otherwise, to republish, to post on servers or to redistribute to lists, requires prior specific permission and/or a fee.

IDPHA 2014, May 20-22

Copyright (c) 2014 ICST 978-1-63190-011-2

DOI 10.4108/icst.pervasivehealth.2014.255433 of independence and life quality of the dementia patient and the carers. Innovative integrated and systemic solutions, i.e. the connection of social, spatial, technical and structural elements, are key to achieve supportive environments for all stakeholders. Such an integrated perspective is the goal of a new interdisciplinary government-funded research program at our university.

A majority of investigations of social, spatial, technical and structural elements in this design space, even in interdisciplinary consortia - as we know from our own experience in EU-projects - are often conducted separately. In the area of ambient assisted living (AAL) a technology push and simultaneous lack of consideration of the social context was observed [5]. Consequently, the AAL-solutions were not perceived as useful resulting in a slow uptake of technologies in practice [4]. For a holistic view on the possibilities of enhancing the care and life quality for people with dementia, interdisciplinarity is deemed crucial. However, there are few guidelines how to successfully implement interdisciplinary design in practice.

From presentations (e.g. publications) of interdisciplinary design work, one could get the feeling that interdisciplinary integration (see definitions below) takes place. However, we are confined to the representations of the design work by their authors, often focusing on the outcomes of the process and high-level descriptions of method, leaving many aspects "black-boxed". What we refer to are factors that lead to one design outcome or another and that define the knowledge created throughout the process and to be potentially applied in other projects (be it methodological or otherwise). These factors, often challenging for the design team and sometimes even invisible to those involved, include (among others) politics of participation, power structures, difficulties arising from disciplinary backgrounds (e.g. language, epistemologies) and choice of materials (digital or not) used. All these may influence the design decisions (i.e. decisions for one alternative and against another) made along the way and ultimately leading to one solution.

In this paper we present some of the challenges we encountered, in the early stages of our interdisciplinary research program, and propose a reflexive approach to mitigate challenges and make visible what is at play in interdisciplinary design - to be discussed at the workshop.

\section{ON INTERDISCIPLINARITY}

To understand the nature (and difficulties) of interdisciplinarity, let's take a closer look on the parts of the word, inter and discipline. By disciplines we refer to academic fields of study. "Each discipline has its own defining elements-phenomena, assumptions, epistemology, concepts, theories, and methodsthat distinguish it from other disciplines." [8] Traditionally, disciplines are categorized broadly into social sciences, natural 
sciences and humanities. Besides these, there are fine and performing arts as well as applied/professional fields.

The prefix "inter" refers to "between, among, in the midst," or "derived from two or more." Commonly, interdisciplinary work deals with "contested spaces", complex problems or issues that are in focus in several disciplines, such as public health systems (with its economic, medical, social and technical dimensions). The attention in interdisciplinary work lies on the problem itself, not the involved disciplines. With interdisciplinarity a more comprehensive understanding of the problem can be achieved through integration, i.e. "a process by which ideas, data and information, methods, tools, concepts, and/or theories from two or more disciplines are synthesized, connected, or blended." [8] Without going into too much detail, we would like to point to a distinction between instrumental and critical interdisciplinarity, which are either problem-driven or society-driven respectively. Where the first offers a pragmatic approach (integrating knowledge from the involved disciplines), the latter also raise epistemological and political questions. Following from these definitions, it should be indisputable that interdisciplinarity is needed to deal with the complex issues around the care and support for people with dementia.

\section{DESIGNING SOLUTIONS FOR PEOPLE WITH DEMENTIA: A NEW RESEARCH PROGRAM}

A range of technologies for dementia care exist that facilitate disease monitoring and personal safety in the home context for people with mild dementia as well as tagging systems for dementia patients with wandering behavior. However, these examples largely represent a surveillance approach rather than the person-centered approach that has been promoted in dementia care for two decades [6]. Examples that have taken a person-centered approach involving people with dementia are still rare (with some exceptions [9, 13]).

In order to design innovative solutions that integrate different perspectives of involved stakeholders as well as social, spatial, technical and structural elements we have recently started to establish an interdisciplinary research team at the University of Applied Science Düsseldorf (see www.nutzerwelten.de) that consists of colleagues from four faculties, i.e. design, media, electrical engineering and social- and cultural studies, as well as external partners from research and practice in ICT, dementia care, architecture and the medical field. Goal of the 4-yearfunded programme is to create strong networks within and outside the university to ensure a user-centered process of designing solutions for people with dementia and their caregivers that can be put into practice. Improving the life quality of all involved in dementia care is the main focus. Therefore, an approach to account for the needs and values of the different stakeholders and actively involving stakeholders in the design of new solutions will be used.

\section{AREAS FOR CONCERN FOR INTERDISCIPLINARY DESIGN}

In this section we present a number of areas of concern or challenges (some encountered in this early phase of our research). This is by no means an exhaustive list and some of the issues have been mentioned elsewhere.

Take the following example. One first project in our research agenda deals with designing (ambient) technologies to support communication between dementia patients and their relatives or (informal) carers. Before going into the field to study people's communication and its problems in-situ, a valid question is 'What is communication?'. Many would agree that communication is not only manifested in verbal exchange of information (speech or writing, where the problem may, e.g., be distance between patient and carer and a potential technology could be a simplified telephone or email interface), but also in other ways, such as behaviors, signals, visuals, emotions etc. Communication may be intentional or unintentional. We experienced ourselves that in different meetings with people from different disciplines, conceptions of what communication is in the context of dementia and which type of communication should be in the focus of our first design case differed (as did the sources drawn on, such as literature, experience from practice). A different conception will lead to a different design outcome, but the question is 'how is it decided in an interdisciplinary process what conception to choose?' While ideally all stakeholders' opinions should be evaluated and costs and benefits traded to design one or more solutions, such a decision may depend on who is present in the design team, at a project meeting, who raises their voices the loudest, who is consulted in workshops and interviews, etc.

\subsection{Power relations}

In one of the meetings mentioned above some people were rather dominantly expressing their opinions, while others remained silent throughout the meeting. If not accounted for, dominant people could easily push through a certain view of the problem at hand and steer the design process in that direction. Some conceptions and definitions of terms, in our case 'communication', may surface, while others may remain unknown. Power relations, perceptions of one's own or others' power, will influence how decisions are made.

\subsection{Language and professional perspective}

Differing from the case presented above, where it was explicitly asked and negotiated what conception of communication would be the focus of the design challenge, there are also occasions when terms are seemingly understood by all people, though unknowingly in different ways (e.g. based on definitions from their own disciplines). Glossaries can help here, but still the people involved first have to identify the words that may have different meanings. There can, however, be a social bias to ask the supposedly obvious or people simply just see the concepts and problems only from their profession. On the other hand (technical) language can also provide safety when someone feels insecure or be used as a tool for power (using specific language may exclude others from a conversation).

\subsection{Roles, Interests and Expectations}

Every person involved in interdisciplinary design plays a certain part in a design activity or situation in the process, i.e. has a role. This role can change and people can have more than one role depending on the situation. This in itself is not a problem, what is important, however, is the understanding of one's role and that of others. As not all partners may have been involved in formulating a project proposal, and new partners/people are brought into the design process after the proposal, their roles are not always clear. In our project meeting some partners openly stated that they were not entirely sure of their role in the project. What was further observed in the meeting was that perceptions of the other partners (e.g. considering their time for involvement) that they used to assign them a role were sometimes not accurate. In addition, motivations and interests (scientific, 
economic, altruistic or otherwise), either people's own or those of the institutions they represent, for taking part in a project may influence the role they take, the expectations they have and the ways in which they may (intentionally try to) steer the designs. Often these are left implicit or are invisible to the participants.

\subsection{Involvement of stakeholders}

As stated earlier, designing solutions for people with dementia should involve people with dementia to ensure person-centered care. Active participation of people suffering from cognitive limitations due to dementia, is, however, not easy and few methods have been developed for co-design with people with dementia (with the notable exception of [13]). However, there is a discrepancy of values of people with dementia (e.g. meaningful social interaction) and their caring relatives (e.g. safety). Given the complex problems that interdisciplinary design intends to solve, many stakeholders exist, raising questions about who to involve, in what stage and how.

\subsection{Long-term effects of design decisions}

Another issue, more generally applicable to pervasive health and innovation, is that we have to be aware that while we create solutions to fit into current care environments, we are changing these environments including their social and professional practices and feelings of people. Our designs may even influence the perspectives of people about concepts such as care or aging. Designing 'assistive' technology could add to stereotypes of seniors being frail and in need, while technology that enhances seniors' skills and empowers them to use their knowledge, may paint a picture of wise elders or fosters the discourse of 'successful aging'. Systems that foster informal care networks may change the view on how care is defined and so on. Whether conscious or not, questions about the ethics of our interventions arise as well as methodological questions about the generation of knowledge that is drawn from socially constructed solutions, that go further than simply a new technical artifact, but also include social innovation and remind us of the complexity of socio-technical networks that are produced here.

\section{OUR CRITIQUE}

The areas for concern identified above may be tacit or known, unintentional or intentional. Consequently, it is difficult to be aware of all the issues at play in interdisciplinary design. It is even questionable whether a design team can be aware of all these throughout the design process. Another problem that arises over time is "black-boxing", i.e. some knowledge is taken for granted, methods become acknowledged and are used without reflection or critical questioning. In many publications in our field, those methods are mentioned but not described in detail. Their applicability and correct execution may be taken for granted. Furthermore, publications focus more on the sociotechnical innovations themselves than on the process of how they were produced. Step-by-step procedures, personal attitudes and feelings, power struggles, failures, or discarded ideas or artifacts are often left inside the black box. This leads to limited visibility for how the process of interdisciplinary design unfolds and how knowledge is created. Consequently, transferring knowledge from one design case to another will be limited.

\section{PROPOSAL: REFLEXIVE PRACTICE IN INTERDISCIPLINARY DESIGN}

Following from our critique we advocate more visibility of the factors discussed earlier, both within the design team throughout the design process and in presentations of interdisciplinary design (be it publications or other forms of representations). In the following we propose a number of frameworks and methods that we deem useful in doing so, and intent to use in our project.

\subsection{Reflection in and on design}

In order to make things visible, we believe the concept of reflection is crucial. In the 'reflective practitioner' Schön [11] introduced two types of reflection: reflection-in-action and reflection-on-action. Reflection-in-action occurs during the completion of a task and helps us reshape that task as we go along. Critical is the concept of knowing-in-action, which is often tacit information about doing something (just like many things in interdisciplinary design teams). According to Schön, reflection-in-action is commonly triggered when a surprise occurs during a task. Such surprises occur because knowledge we transfer from one situation to another does not fit. Through reflection we can adjust our knowledge to the current task.

Reflection-on-action occurs after we finished a task, when we evaluate our process. "We reflect on action, thinking back on what we have done in order to discover how our knowing-inaction may have contributed to an unexpected outcome" [11, p. $26]$. Both concepts are useful, but hard to put into practice without long-term experience in the doing interdisciplinary design. Let us therefore, look at ways to support reflection.

\subsection{Supporting reflection and 'making visible'}

Some of the areas of concern described above, such as reflecting on which stakeholders to involve in the design process as well as on the long-term systemic effects of new technologies, can be accounted for by using a value sensitive design (VSD) approach [2]. VSD introduces the concepts of indirect stakeholders (nonusers, but still affected by the technology) and investigates longterm systemic effects that technology has on the quality of life of all stakeholders, thereby shifting the view from the current technical solution to envisioning the future socio-technical changes, that are either desirable or should be hindered. VSD is pro-active in the sense that it builds 'checks' into the design process to reflect on how designs are created and which effects they may have. A method inspired by Schön's theory of reflective practice is the value-sensitive action reflection model [14], a tool using prompts (either created by designers and stakeholders) in a co-design session with stakeholders to reflect "the particular socio-technical setting of use ... and more generally the social and contextual issues that are easily overlooked." While VSD provides a toolbox for design, it cannot make visible all factors discussed earlier.

The complexity and heterogeneity of interdisciplinary design in pervasive health demands an additional reflexive evaluation. To analyze the dynamics of the process, the power relations at play, the agency of artifacts, the self in the design team and generally making the tacit visible, an analytical framework that accompanies design processes reflexively becomes necessary. Actor-Network Theory (ANT, e.g. [10]) offers this reflexive character by providing a rich repertoire of theoretical concepts including the notions of translation, generalized symmetry and the heterogeneous network to understand socio-technical 
processes and gaining a closer look to the different modes of interaction in the design process and their relational character. ANT is given a suitable methodological and theoretical background not only to engage social anthropologists in a primarily techno-scientific field but also to focus on seniors' actual needs. By focusing on the social practices of the designers, the interaction between designers, designed objects and the imagined or real users and stakeholders as well as on the different levels of generating and translating knowledge in these various interactions, an ethnographic perspective can bring a new quality to the design. "Only by describing both the production task and the hidden task in articulation, together and recursively, can we come up with good analysis of why some systems work and others do not." [12, p.387]. We are, therefore, proposing a constellation where the ethnographer becomes part of the interdisciplinary design team as an active observer. By discussing the observed practices regularly we establish a reflexive part in the design process that is challenging as well as deepens the understanding of the political character of design and its implementation on society. Taking the own visibility as an active observer seriously also provides one means of "making things public" [7].

In our research program a first project on designing technologies to support communication for people with dementia will serve as a test-bed for this collaboration between a social ethnographer (second author) and the design team (represented by the first author).

\subsection{Presenting interdisciplinary design}

As this workshop shows, it is important for those involved in interdisciplinary design to share their knowledge beyond stating the methods and outcomes, but to share experiences of how the design process unfolded, what barriers were met and what lessons were learned. Besides coming together and discussing these aspects face-to-face other means for more visibility of the design process can be found. First, publication formats may be changed to allow for unpacking the black box. Just recently DIS, a leading design conference, introduced pictorials - as "new opportunities ... to communicate design practices, processes, products and artifacts to the HCI community" (Call for Paper). In pictorials authors are encouraged to describe design decisions that affected elements of prototypes, successful as well as failed attempts, lessons learned, deployments of artifacts and generally insights often unmentioned. Such descriptions could be given and be enhanced through design sketches, annotated images, illustrations and diagrams, field notes or sketches, or collages of images. Besides such visual and commonly unmentioned material from the design process, the style of presenting interdisciplinary design could follow that of thick descriptions [3], to not simply describe and explain (e.g. a technology or human behavior), but also to bring the heterogeneous context into focus, so that it becomes meaningful for both the insider as well as the outsider. The context includes the perspectives and attitudes of the authors, who 'translate' their observations in the writing. These presentations allow for critical reflection on the presented interdisciplinary designs by the readers.

\section{CONCLUSION}

We presented a number of areas for concern for interdisciplinary design of solutions for people with dementia and their caregivers, some of them experienced first-hand in a recently started research program. We have critiqued the lack of visibility of factors at play that may influence the creation of knowledge and design solutions in this field. Further, we proposed an approach to make such factors more visible through reflective practices based on VSD and ANT - an approach we are currently investigating in our research program.

\section{ACKNOWLEDGEMENTS}

This research is made possible through the funding of the $\mathrm{FH}$ STRUKTUR program of the government of North RhineWestphalia.

\section{REFERENCES}

[1] Alzheimer Disease International. 2010. World Alzheimer Report:www.alz.co.uk/research/files/WorldAlzheimerRepo rt2010.pdf.

[2] Friedman B., Kahn P. H., Jr., and Borning, A. Value sensitive design and information systems. In Humancomputer Interaction in Management Information Systems. Foundations, M.E. Sharpe, Armonk, NY, 2006, 348-372.

[3] Geertz, C. Thick description: toward an interpretive theory of culture, in: The interpretation of cultures: selected essays. New York, N.Y. (1973): Basic Books, pp. 3-30.

[4] Greenhalgh, T., Procter, R., Wherton, J., Sugarhood, P., and Shaw, S. The organising vision for telehealth and telecare: discourse analysis. BMJ Open 2:e001574 (2012).

[5] Huang J.C. Exploring the Acceptance of Telecare Among Senior Citizens: An application of back propagation network. Telemedicine and e-Health 17, 2 (2011) 111-117.

[6] Kitwood, T., \& Bredin, K. (1992). Towards a theory of dementia care: Personhood and well being. Ageing and Society, 12, 269-287.

[7] Latour, B. and Weibel, P. (2005). Making things public, atmospheres of democracy. Cambridge: MIT Press.

[8] Repko, A.F. Interdisciplinary Research: Process and Theory. Sage Publications, 2012.

[9] Robinson,L., Brittain, K., Lindsay,S., Jackson,D. and Olivier, P. Keeping in touch everyday (KITE) project developing assistive technologies with people with dementia and their carers to promote independence. International Psychogeriatrics (2009), 21:3, 494-502.

[10] Tatnall, Arthur, Anthony Gilding, 'Actor-Network Theory and Information Systems Research', in: Proc. 10th Australasian Conference on Information Systems, 1999, pp. 955-966.

[11] Schön, D.A. 1983. The Reflective Practitioner: How Professionals Think in Action. Basic Books, New York.

[12] Star, S. L. (1999). The Ethnography of Infrastructure. American Behavioral Scientist, 43, p. 377-391.

[13] Wallace, J., McCarthy, J., Wright, P.C. and Olivier, P. 2013. Making design probes work. In Proceedings of the SIGCHI Conference on Human Factors in Computing Systems (CHI '13). ACM, New York, NY, 3441-3450.

[14] Yoo, D., Huldtgren, A., Woelfer, J.P., Hendry, D.G. and Friedman, B. 2013. A value sensitive action-reflection model: evolving a co-design space with stakeholder and designer prompts. In Proceedings of the SIGCHI Conference on Human Factors in Computing Systems (CHI '13). ACM, New York, NY, USA, 419-428. 